\title{
Systolic Hypertension Mechanisms: Effect of Global and Local Proximal Aorta Stiffening on Pulse Pressure
}

\author{
Philippe Reymond, ${ }^{1}$ Nico Westerhof, ${ }^{2}$ and Nikos Stergiopulos ${ }^{1}$ \\ ${ }^{1}$ Laboratory of Hemodynamics and Cardiovascular Technology, Ecole Polytechnique Fédérale de Lausanne, EPFL STI IBI-STI \\ LHTC, BM 5128, Station 17, 1015 Lausanne, Switzerland; and ${ }^{2}$ Department of Pulmonary Diseases, VU University Medical \\ Center, Amsterdam, The Netherlands
}

(Received 9 June 2011; accepted 8 October 2011; published online 21 October 2011)

Associate Editor Jane Grande-Allen oversaw the review of this article.

\begin{abstract}
Decrease in arterial compliance leads to an increased pulse pressure, as explained by the Windkessel effect. Pressure waveform is the sum of a forward running and a backward running or reflected pressure wave. When the arterial system stiffens, as a result of aging or disease, both the forward and reflected waves are altered and contribute to a greater or lesser degree to the increase in aortic pulse pressure. Two mechanisms have been proposed in the literature to explain systolic hypertension upon arterial stiffening. The most popular one is based on the augmentation and earlier arrival of reflected waves. The second mechanism is based on the augmentation of the forward wave, as a result of an increase of the characteristic impedance of the proximal aorta. The aim of this study is to analyze the two aforementioned mechanisms using a 1-D model of the entire systemic arterial tree. A validated 1-D model of the systemic circulation, representative of a young healthy adult was used to simulate arterial pressure and flow under control conditions and in presence of arterial stiffening. To help elucidate the differences in the two mechanisms contributing to systolic hypertension, the arterial tree was stiffened either locally with compliance being reduced only in the region of the aortic arch, or globally, with a uniform decrease in compliance in all arterial segments. The pulse pressure increased by $58 \%$ when proximal aorta was stiffened and the compliance decreased by $43 \%$. Same pulse pressure increase was achieved when compliance of the globally stiffened arterial tree decreased by $47 \%$. In presence of local stiffening in the aortic arch, characteristic impedance increased to $0.10 \mathrm{mmHg} \mathrm{s} / \mathrm{mL}$ vs. $0.034 \mathrm{mmHg} \mathrm{s} / \mathrm{mL}$ in control and this led to a substantial increase $(91 \%)$ in the amplitude of the forward wave, which attained $42 \mathrm{mmHg}$ vs. $22 \mathrm{mmHg}$ in control. Under global stiffening, the pulse pressure of the forward wave increased by $41 \%$ and the amplitude of the reflected wave by $83 \%$. Reflected waves arrived earlier in systole, enhancing their contribution to systolic pressure. The effects of local vs.
\end{abstract}

Address correspondence to Philippe Reymond, Laboratory of Hemodynamics and Cardiovascular Technology, Ecole Polytechnique Fédérale de Lausanne, EPFL STI IBI-STI LHTC, BM 5128, Station 17, 1015 Lausanne, Switzerland. Electronic mail: philippe. reymond@epfl.ch global loss of compliance of the arterial tree have been studied with the use of a 1-D model. Local stiffening in the proximal aorta increases systolic pressure mainly through the augmentation of the forward pressure wave, whereas global stiffening augments systolic pressure principally though the increase in wave reflections. The relative contribution of the two mechanisms depends on the topology of arterial stiffening and geometrical alterations taking place in aging or in disease.

Keywords-Wave reflection, Characteristic impedance, Arterial compliance, Forward pressure wave, Reflected wave.

\section{INTRODUCTION}

Loss in total systemic compliance leads to an increase in pulse pressure (PP) and, in consequence, to an augmentation of systolic pressure. This was proven by a large number of studies in animals ${ }^{5,12}$ and in the human. ${ }^{1}$ The increase in PP, when compliance decreases, can be attributed to loss in Windkessel function. ${ }^{1,15-17}$ The Windkessel model, however, does not account for wave propagation phenomena, which also play an important role in the development of systolic hypertension following arterial stiffening. The prevailing theory is that aortic stiffening leads to an increase in wave speed and in the amplitude of the reflected wave. The augmented reflected wave will therefore arrive at the ascending aorta earlier, during late systole, and it will be added to forward running wave leading to a substantial increase in systolic pressure. ${ }^{11}$ This mechanism is evidenced by the characteristic change in the shape of the proximal aortic wave (type A in stiff/aged aortas vs. type C in young/ elastic aortas), see Murgo et al. ${ }^{10}$ and by an increase in the augmentation index (AI), which is non-dimensional wave shape index reflecting the relative contribution of the reflected waves on the pulse pressure. Numerous 
clinical studies have shown an increase in $\mathrm{AI}$ in presence of aortic stiffening and in aging.

A second mechanism by which aortic stiffening contributes to systolic hypertension has been proposed. ${ }^{9}$ The underlying theory is that aortic stiffening would lead to an increase in the characteristic impedance of the proximal aorta, which means a proportional increase in the forward running pressure wave, if cardiac output is maintained. The characteristic impedance of the proximal aorta is estimated as $Z_{\mathrm{c}}=\rho \cdot c / A$, where $\rho$ is blood density, $c$ the local wave speed, and $A$ the local cross-sectional area of the aorta. Hence, an increase in characteristic impedance of the proximal aorta can result from aortic stiffening (increase in wave speed $c$ ) but also from lower aortic diameter; hence arterial geometry is also thought to be a determining factor.

The two aforementioned mechanisms are distinctly different. The former is focusing on the effects of the reflected waves, whereas the latter is based on the augmentation of the forward wave. A careful look at the respective mechanisms shows that the former is sensitive to the increase in wave speed along the entire aortic trunk, whereas the latter it is based principally on the amplification of the forward pressure wave as the heart injects into a stiff proximal aorta. The goal of our present work is to analyze the two mechanisms by simulating the effects of aortic stiffening on aortic pressure using a 1-D model of the arterial tree. In order to do so we have considered aortic stiffening in two distinctly different manners: first, aorta is stiffened only locally in the proximal aorta region, thereby increasing the characteristic impedance of the aorta while at the same time decreasing total arterial compliance and, second, by stiffening uniformly the entire arterial tree.

\section{METHODS}

\section{Brief Description of the 1-D Model of the Arterial Circulation}

The 1-D model of blood flow in the arterial trees solves the integrated form of the momentum and continuity equations applied over each arterial segment. A non-linear viscoelastic constitutive law for the arterial wall was considered. The intimal shear stress and nonlinear convective acceleration terms are modeled using the Witzig-Womersley theory. All distal vessels are terminated with three-element Windkessel models to account for the resistance, $R_{\mathrm{T}}$, and compliance, $C_{\mathrm{T}}$, of the distal vascular beds. Total compliance is the sum of volume compliance of the arterial segments included in the 1-D model and the compliance of the terminal Windkessel. A detailed description of the mathematical formulation including governing equations and boundary conditions is presented in Reymond et al. ${ }^{14}$

This generic arterial tree model has been validated quantitatively with in vivo measurements performed and averaged on groups of 6 and 8 subjects with MRI and Doppler techniques, respectively. In a second study involving the same 1-D formulation, a patientspecific model was validated, giving further proof that the 1-D models can provide accurate predictions of pressure and flow in the entire systemic circulation. ${ }^{13}$

At its proximal end (root of the ascending aorta), the arterial tree was coupled to a model of the left ventricle based on the varying elastance model (VEM). ${ }^{2,3}$ Same VEM parameters (normalized elastance curve, maximum elastance, and time to reach maximum elastance were utilized for all simulations.

\section{Local Proximal Aorta Stiffening}

The characteristic impedance, $Z_{\mathrm{c}}$, characterize the proportionality of pressure, $P$, to the flow, $Q$, in the early systole and in absence of wave reflection ${ }^{7}$ :

$$
Z_{\mathrm{c}}=\frac{\Delta P}{\Delta Q}
$$

$Z_{\mathrm{c}}$ may be estimated from the local area compliance $\left(C_{\mathrm{A}}=d A / d P\right)$

$$
Z_{\mathrm{c}}=\sqrt{\frac{\rho}{A C_{\mathrm{A}}}}
$$

where $\rho$ is the blood density, and $A$ the cross-sectional area of the lumen.

We chose to increase the characteristic impedance by decreasing only the distensibility, $D_{\mathrm{w}}=C_{\mathrm{A}} / A$ of the proximal aorta (segments 1-95-2-14-18-27 Fig. 1) by a factor 10 , by changing the elastic modulus, without changing the aortic dimensions. The remaining arterial tree segments kept the same elastic properties as the generic tree (control case). Terminal compliances, $C_{\mathrm{T}}$, at distal ends were also conserved. However, due to the decrease of the arterial compliance, its contribution to the total compliance increased. Distal resistances, $R_{\mathrm{T}}$, however, were increased by $+21 \%$ to obtain the same diastolic pressure as the control case. MAP was increased by $11 \%$. This was done in accordance to the results of in vivo studies, where acute decrease in compliance was always accompanied by increase in peripheral resistance as to preserve diastolic pressure and, in consequence, coronary perfusion. ${ }^{4,5}$

\section{Global Stiffening of the Arterial Tree}

Global stiffening was achieved by decreasing distensibility of all arterial segments by $40 \%$. The level of decrease in distensibility was found by trial and error, 


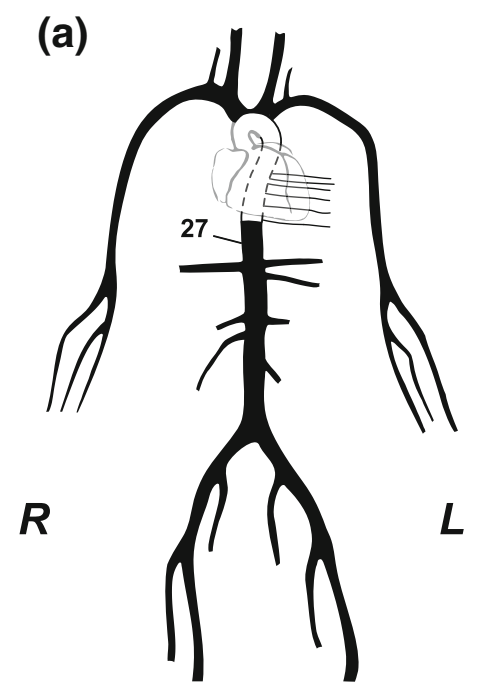

(b)

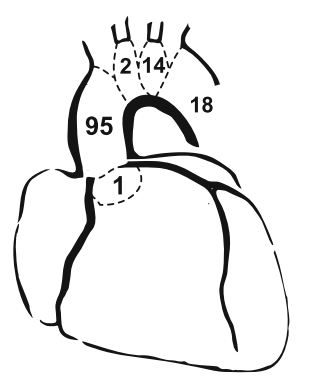

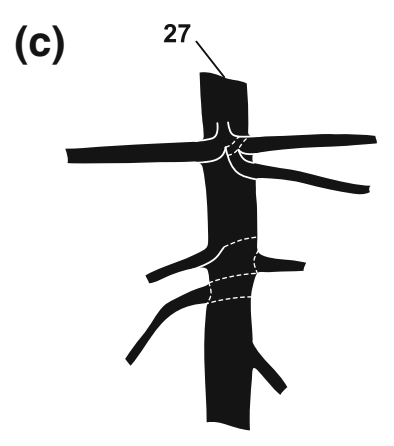

$L$

FIGURE 1. Schematic representation of the arterial tree, adapted from Reymond et al. ${ }^{14}$ (a) Main systemic arterial tree, (b) detail of the aortic arch, (c) detail of the principal abdominal aorta branches.
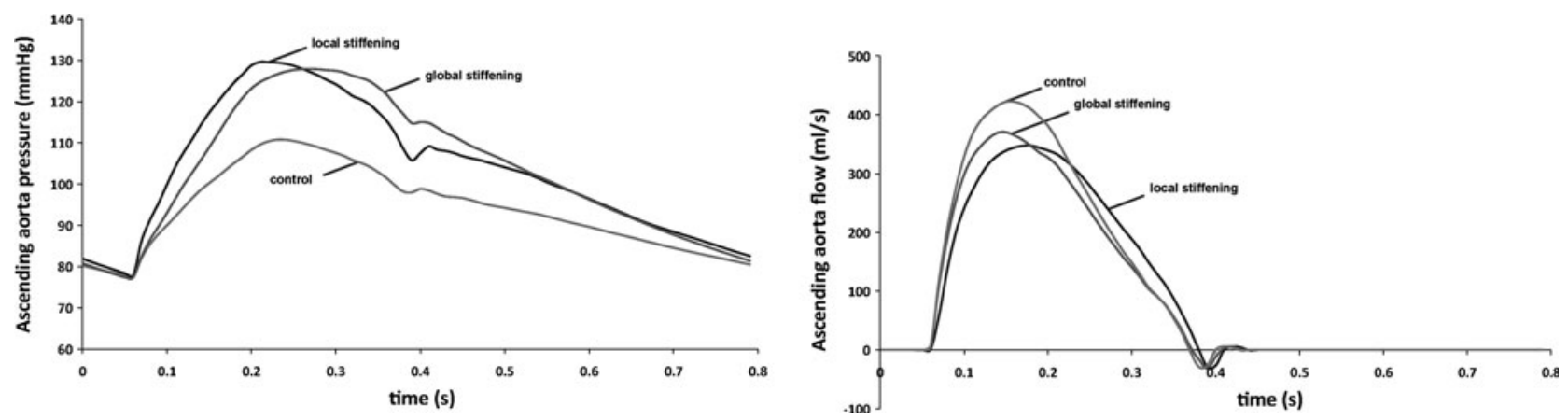

FIGURE 2. Pressure (left) and flow (right) waveforms at the proximal ascending aorta level. They are drawn for the control case, local and global stiffening of the arterial tree.

the criterion being the same proximal aorta pulse pressure as the one obtained with local stiffening. Distal Windkessel compliances, $C_{\mathrm{T}}$, were decreased in proportion to keep their relative contribution to the total compliance the same as for the control case. Distal resistances, $R_{\mathrm{T}}$, were also adapted $(+25 \%)$ to keep diastolic pressure as the control case, as was done for the case of local stiffening. MAP increased by almost the same amount as for local stiffening. On the same token, global stiffening lead to similar decrease in total arterial compliance as for local stiffening ( $-47 \%$ vs. $-43 \%$, respectively), which is an expected result since both stiffening approaches were forced to yield the same pulse pressure and the cardiac output did not very much $(90.8 \mathrm{~mL} / \mathrm{s}$ for local stiffening vs. $88.3 \mathrm{~mL} / \mathrm{s}$ for global stiffening).

\section{Analysis}

The characteristic impedance at the root of the ascending aorta is estimated as the average value of the modulus of the input impedance in the frequency range of 4-10 Hz. Total pressure waves were separated into forward, $P_{\mathrm{f}}$, and backward (reflected), $P_{\mathrm{b}}$, wave components using the formulas ${ }^{19}$ :

$$
\begin{aligned}
& P_{\mathrm{f}}=\frac{P+Z_{\mathrm{c}} Q}{2}, \\
& P_{\mathrm{b}}=\frac{P-Z_{\mathrm{c}} Q}{2}
\end{aligned}
$$

\section{RESULTS}

Figure 2 shows pressure and flow waveforms at the proximal ascending aorta for the control case as well as for the case of local and global stiffening. Important hemodynamic parameters are given in Table 1. Cardiac output, systolic, mean and pulse pressure at the root of the ascending aorta are reported. Compliances are computed based on the compliance-pressure curves, i.e., on model parameters. Total compliance was obtained by addition of all local compliances. 
TABLE 1. Hemodynamic values of the 1-D model simulations for the control case, local aortic stiffening, and global arterial tree stiffening.

\begin{tabular}{lcll}
\hline & $\begin{array}{c}\text { Original arterial } \\
\text { tree (control) }\end{array}$ & $\begin{array}{l}\text { Local proximal } \\
\text { aorta stiffening }\end{array}$ & $\begin{array}{l}\text { Global arterial } \\
\text { tree stiffening }\end{array}$ \\
\hline $\mathrm{PP}(\mathrm{mmHg})$ & 33 & $52(+58 \%)$ & 51 \\
$\mathrm{MAP}(\mathrm{mmHg})$ & 94 & $103.6(+10 \%)$ & 103.3 \\
$\mathrm{PP}_{\text {forward }}(\mathrm{mmHg})$ & 22 & 42 & 31 \\
$\mathrm{PP}_{\text {backward }}(\mathrm{mmHg})$ & 12 & 16 & 22 \\
$\mathrm{PP}_{\text {forward }} / \mathrm{PP}_{\text {backward }}(-)$ & 1.83 & 2.63 & 1.41 \\
$\mathrm{PWV}$ foot to foot; from asc. aorta & 4.1 & 5.3 & 5.0 \\
$\quad$ to femoral artery $(\mathrm{m} / \mathrm{s})$ & 98.5 & 90.8 & 88.3 \\
Cardiac output $(\mathrm{mL} / \mathrm{s})$ & 1.33 & $0.76(-43 \%)$ & $0.70(-47 \%)$ \\
Total compliance $(\mathrm{mL} / \mathrm{mmHg})$ & 1.04 & 0.47 & 0.55 \\
Arterial compliance $(\mathrm{mL} / \mathrm{mmHg})$ & $0.29(22 \%)^{*}$ & $0.29(38 \%)^{*}$ & $0.15(21 \%)^{\star}$ \\
Distal compliance $(\mathrm{mL} / \mathrm{mmHg})$ & 0.034 & $0.10(+194 \%)$ & $0.042(+24 \%)$ \\
$Z_{\mathrm{c}}$ (mmHg s/mL) & & & \\
\hline
\end{tabular}

PP, pulse pressure; MAP, mean arterial pressure; $Z_{c}$, characteristic impedance. Relative differences are referred to the control. Distal compliance is the compliance of all the distal small arteries, arterioles that are modeled with Windkessel and

${ }^{*}$ is the relative contribution of distal compliance to total compliance.
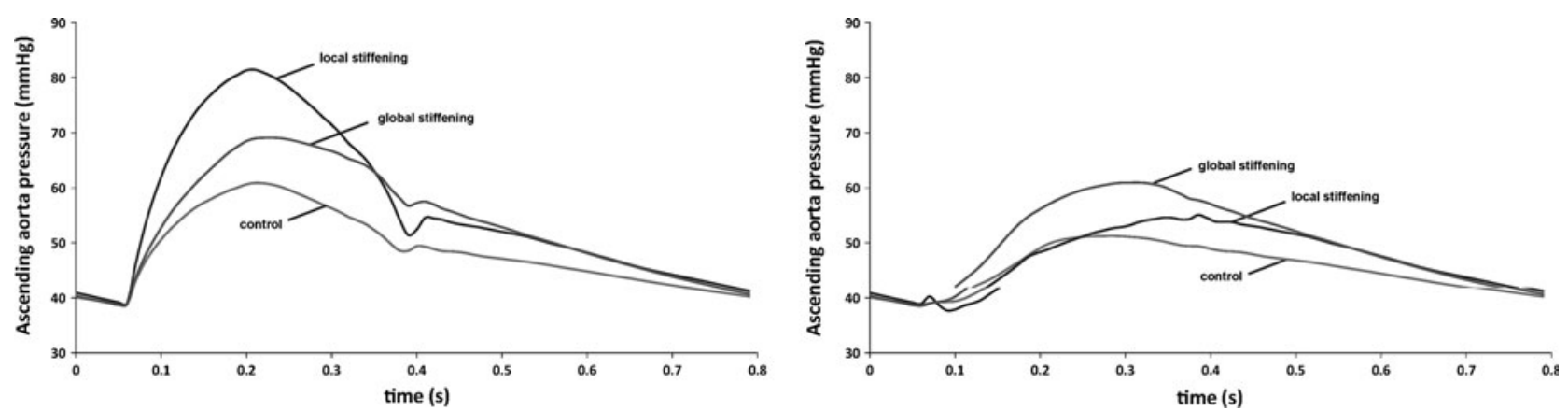

FIGURE 3. Pressure wave separation. Forward (left) and backward (right) pressure wave components at the root of the ascending aorta level. Give details that in total stiff and local stiff, $P_{f}$ is different.

\section{Whole System and Proximal Aortic Stiffening Increase Pulse Pressure Through Different Mechanisms}

Pulse pressure increased by $58 \%$ in presence of a stiff proximal aorta. Total compliance decreased by $43 \%$. Distal compliance was the same as for the control case in absolute value. Characteristic impedance presents the highest change and reached almost 3 times the control value. For the whole stiffening of the arterial tree, distal compliance was decreased by almost a factor $2(0.29-0.15 \mathrm{~mL} / \mathrm{mmHg})$ compared to the control case, to keep its contribution to the total compliance around $21-22 \%$, as it was in the control simulation. In contrast to local stiffening, characteristic impedance increased moderately $(+24 \%)$ compare to the control value.

\section{Analysis of Forward and Backward (Reflected) Waves}

The forward and backward components are presented in Fig. 3, for the control case as well as the case of local and global stiffening. In presence of a locally stiff proximal aorta, it is the forward wave that is substantially amplified (amplitude of forward wave $42 \mathrm{mmHg}$, vs. 22 for control, Fig. 3, left). In contrast, the reflected wave is not amplified in proportion to the forward wave (amplitude of reflected wave $16 \mathrm{mmHg}$ vs. 12 in control, Fig. 3, right). Furthermore, the time of arrival of the reflected wave is practically unaltered and its contribution to early systolic pressure is essentially the same as in control (compare ascending part of the reflected wave between control and local stiffening, Fig. 3, right). Hence, it is quite clear that the main reason for the increase in PP in presence of local stiffening of the ascending aorta is the amplification of the forward wave.

As clearly seen in Fig. 3, the forward wave is amplified by $41 \%$ but at the same time the reflected wave is also amplified by $83 \%$ with respect to control. Furthermore, as seen in Fig. 3, right, the amplified reflected wave arrives in the root of the ascending aorta earlier, thereby contributing more to early systolic pressure. It is therefore clear that in a globally stiff 
aorta the reflected wave is a major contributor to the augmentation of systolic and pulse pressure.

\section{Aortic Input Impedance}

The aortic impedance amplitude and phase have been computed. We observed higher characteristic impedance under local stiffening. Also, the modulus of the input impedance at low frequencies (1st and 2 nd harmonic) is similar for the locally and globally stiffened arterial tree. This is consistent with the fact that these impedance modulus are principally determined by the global "Windkessel" characteristics, i.e., total compliance, which is similar in both arterial trees.

\section{DISCUSSION}

The fact that arterial stiffening, as a result of aging or arterial disease, leads to increase in pulse pressure and systolic hypertension is widely accept and support by numerous clinical studies. The exact mechanism by which this happens is still a controversial topic. ${ }^{18}$ Perhaps the most widely accepted line of thinking is that arterial stiffening increases wave speed and thus reflected waves, often larger in amplitude, arrive earlier in systole adding themselves to the forward wave, thereby augmenting pulse pressure and systolic pressure. ${ }^{11}$ The presence of a significant reflected wave in early systole is often seen in pressure recordings as a pronounced late systolic peak, usually quantified by means of the AI, defined as the ratio of the amplitude of the late systolic peak to pulse pressure. A large number of studies have shown the link between arterial stiffness and AI, contributing thus to a wider use of AI as a useful non-dimensional index relating systolic hypertension to arterial properties (stiffening) and to physiological (i.e., aging) or pathological (i.e., hypertension, arteriosclerosis) processes affecting wave speed and reflections.

A different line of thinking has been recently proposed, according to which systolic hypertension could be primarily due to amplification of forward waves when the heart ejects into a stiff proximal aorta, rather than to augmentation and earlier arrival of reflected waves. Mitchell et al. ${ }^{9}$ performed noninvasive pressure and flow measurements on subjects with systolic hypertension, and concluded that systolic hypertension is primarily due to an increase in wall stiffness and reduced aortic diameter rather than early wave reflection. Indeed, an increase in ascending aortic stiffness or a decrease in its diameter would both lead to an increase in characteristic impedance, in accordance to $Z_{\mathrm{c}}=\rho \cdot c / A, c$ being the local wave velocity. By definition, characteristic impedance is equal to the ratio of pulsatile pressure to pulsatile flow in absence of reflection $\left(Z_{\mathrm{c}}=\Delta p / \Delta Q\right)$. Hence in early systole, where reflections have not yet arrived from the periphery, the pressure increase $\Delta p$ in the early systolic phase will be essential equal to $\Delta p$ of the forward wave and by virtue of the equation defining characteristic impedance $\Delta p=Z_{\mathrm{c}}{ }^{*} \Delta Q$. If cardiac output is maintained so that $\Delta Q$ is no affected by stiffening, then the up rise in pressure in early systole will be proportional to the increase in characteristic impedance and a major contributor to systolic hypertension.

We find that both mechanisms have their merit because they are based on physically sound principles. We hypothesized that the relative importance of each mechanism depends on the "topology" of arterial stiffening or geometrical alterations taking place in aging or disease. To elucidate this point we have considered and simulated using a realistic model of the arterial tree two rather extreme cases: one in which arterial stiffening is uniformly applied to all arterial segments and peripheral beds (global stiffening) and one in which stiffening is applied only to the proximal aorta region (local stiffening). The simulations and analysis of the results showed in a rather clear and direct way the anticipated differences in the two mechanisms contributing to systolic hypertension.

Under global stiffening, both the forward and backward running waves are augmented in amplitude.

The relative increase in the amplitude of the forward wave is $41 \%$, which, in part at least, is due to the increase in characteristic impedance of the proximal aorta $(+24 \%)$. The amplitude of reflected wave is increased by $83 \%$, indicating an increase in wave reflection coefficient by $30 \%$. Our analysis shows that the increase in the reflected wave amplitude is primarily due to changes in compliance and terminal resistance in the distal reflection sites. The arrival of reflected waves earlier in systole is clearly shown in Fig. 3, right. As seen in Fig. 3 and concluded earlier, in presence of global stiffening the reflected waves do contribute to systolic hypertension, although the amplification of the forward wave is also an important contributor as well.

When we stiffened the proximal aorta only in a manner as to attain the same pulse pressure as for global stiffening, the amplitude of forward wave was amplified by $91 \%$, whereas the amplitude of the reflected wave was increased only by $33 \%$, indicating a ratio of backward to forward wave amplitude of 0.38 , which is actually lower than control (0.55). It is likely that reflected waves traveling backwards along the thoracic aorta are re-reflected at the distal end of the stiff aortic arch, thus never reaching the root of the ascending aorta. We applied the wave transmission theory to calculate the reflection coefficient of the 
backward wave returning from the abdominal aorta at the interface with the stiff proximal aorta. The reflection coefficient is calculated to be 0.43 , which is much higher than the reflection coefficient in case of the globally stiffened arterial tree, namely -0.21 . Hence, in theory at least, reflected waves under local stiffening will have harder time reaching the proximal aorta.

In our numerical simulations, stiffening of the proximal aorta led to a 3 -fold increase in characteristic impedance, a $43 \%$ decrease in total compliance with a distal compliance maintained constant (its contribution to the total compliance increased from 22 to $38 \%$ ) and a $58 \%$ increase in pulse pressure.

These results agree well, at least in a qualitative sense, with the in vivo experiments by Ioannou et al. ${ }^{4,5}$ Ioannou et al. placed a non-compliant Dacron sleeve around the aortic arch of swines, thereby decreasing substantially the local aortic compliance. Two days after the operation, they reported an increase in PP by $86 \%$ and a decrease in total arterial compliance by $50 \%$ while the characteristic impedance of the proximal aorta increased to 2.5 times its control value. Ioannou et al. also showed that the increase in pulse pressure was mainly attributed to the large increase $(+96 \%)$ in the forward wave. Both studies confirm that stiffening of the proximal aorta only has a major impact on the total arterial compliance, providing further support on the commonly accepted fact that most of the total systemic compliance resides in the proximal aorta region. ${ }^{16}$

It is of interest to note that in both local and global stiffening a similar decrease in total arterial compliance ( $-43 \%$ in local vs. $-47 \%$ in global stiffening) led to a similar increase in PP $(52 \%$ in local vs. $51 \%$ in global stiffening). The decrease in compliance under local and global stiffening lead to an increase in PWV estimated from the foot-to-foot transit time between the ascending aorta and the iliac bifurcation. However, the increase was $29 \%$ for the local stiffening and somewhat lower for the globally stiff arterial tree $(+22 \%)$. The difference may be attributed to the fact that in the case of local stiffening we affect solely the aorta and thus the aorto-iliac path is stiffer than in the case of global stiffening. We observe also that although both types of stiffening lead to the same increase in PP, the pressure wave shapes are different. So despite distinctly different effects on the forward and back running wave components the loss in compliance appears to lead to the same level of PP increase. This seems to suggest that, overall and by enlarge, what determines the increase in PP is the loss in compliance and the exact topology defining where the decrease in compliance takes place (local vs. uniformly distributed along the entire arterial network) plays a secondary role. In other words, the Windkessel effect prevails and defines PP for a given ejection volume. This was exactly the finding of Chemla et al. ${ }^{1}$ and the conclusion of Stergiopulos and Westerhof. ${ }^{17}$ Stergiopulos and Westerhof showed that, for a given ejection volume, PP can be completely and very precisely determined by only two arterial parameters: total arterial resistance, $R$, and total arterial compliance, $C$. The explanation is the PP is primarily determined by the low frequency components of the pulse (first 3 to 4 harmonics) and for these frequencies the 2-element Windkessel provides and very faithful description of the input impedance of the arterial system. ${ }^{15}$ This seems to be a paradoxical finding, because the Windkessel model neglects wave phenomena, which apparently play an important role in shaping aortic pressure and augmenting systolic pressure in presence of stiffening. Yet the Windkessel model predict PP in a very precise manner and that in presence

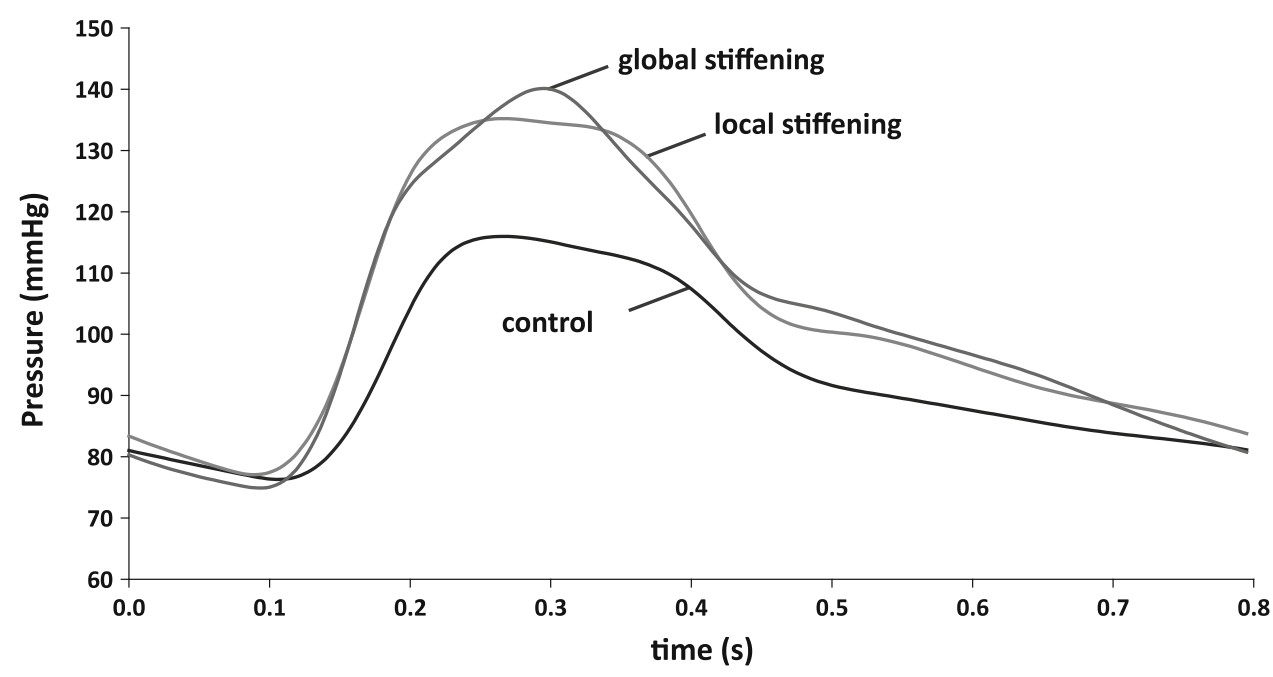

FIGURE 4. Pressure waveforms at the left brachial artery, for the control, the local and global stiffened arterial trees. 
or absence of strong reflections, ${ }^{17}$ however, falls short in describing detailed morphology and cannot take thoroughly into account changes in local stiffness.

\section{Distal Pressure Waveforms}

Brachial artery pressure is monitored and of particular clinical interest, therefore pressure waveforms of the control, local, and global stiffening at the brachial level are presented in Fig. 4. The systolic pressure under global stiffening is $5 \mathrm{mmHg}$ higher than under local stiffening, whereas such a difference does not exist in the corresponding proximal aorta pressure curves. Differences between aortic and brachial pressures are dependent on factors influencing wave propagation characteristics and have been reported in the literature.

We also studied the pressure waveforms in the infrarenal abdominal aorta. This location is situated distally of the stiff region of the thoracic aorta. We observe that the two pressure curves under local or global stiffening are fairly similar in terms of both amplitude and shape.

\section{Limitation}

We have limited our simulations to changes in arterial compliance and we have not touched upon changes in geometry. From a theoretical standpoint, changes in geometry will also impact on wave transmission properties and will affect wave speed and characteristic impedances. Mitchell et al. have pointed out the possibility that systolic hypertension in aging may very well be due to an increase in characteristic impedance not only because of stiffening but also due to decreased aortic diameter. ${ }^{8,9}$ Arterial dimensions affect also wave reflections. An increase of reflection coefficient that may occur with age due to mismatch of cross-sectional geometries at the thoracic-abdominal location, has been suggested by Langewouters et al. based on ex vivo measurements on cadaveric human aortas. ${ }^{6}$ Other studies have pointed to changes in aortic dimension with aging which entrain important changes in the wave reflection intensity and profile. ${ }^{20}$ The topic is of importance and warrants further investigation.

\section{CONCLUSION}

In summary, the impact of local stiffening of the proximal aorta and global uniform stiffening of the arterial tree was modeled and analyzed by means of a 1-D model of the human systemic circulation. When proximal aorta and global stiffening led to the same reduction in total arterial compliance then the increase in aortic pulse pressure was also similar, however, the mechanisms by which this increase in pulse pressure took place are different. Local proximal aorta stiffening induces an increase in the characteristic impedance and, in consequence, an increase in the amplitude of the forward pressure wave. Global stiffening increased the amplitude and reduced the travel time of the reflected waves, which by arriving back to the ascending aorta in early systole become major contributors to the increase in systolic pressure. We believe that the two mechanisms coexist and their importance in aging and in presence of different pathologies needs to be carefully addressed in future studies.

\section{ACKNOWLEDGMENTS}

This work was supported in part by the European Commission integrated program IST-027703 (@neurIST Project).

\section{REFERENCES}

${ }^{1}$ Chemla, D., J. L. Hebert, C. Coirault, K. Zamani, I. Suard, P. Colin, and Y. Lecarpentier. Total arterial compliance estimated by stroke volume-to-aortic pulse pressure ratio in humans. Am J Physiol 274:H500-H505, 1998.

${ }^{2}$ Fitchett, D. H. LV-arterial coupling: interactive model to predict effect of wave reflections on LV energetics. Am J Physiol 261:H1026-H1033, 1991.

${ }^{3}$ Formaggia, L., D. Lamponi, M. Tuveri, and A. Veneziani. Numerical modeling of 1D arterial networks coupled with a lumped parameters description of the heart. Comput. Methods Biomech. Biomed. Eng. 9:273-288, 2006.

${ }^{4}$ Ioannou, C. V., D. R. Morel, A. N. Katsamouris, S. Katranitsa, I. Startchik, A. Kalangos, N. Westerhof, and N. Stergiopulos. Left ventricular hypertrophy induced by reduced aortic compliance. J. Vasc. Res. 46:417-425, 2009.

${ }^{5}$ Ioannou, C. V., N. Stergiopulos, A. N. Katsamouris, I. Startchik, A. Kalangos, M. J. Licker, N. Westerhof, and D. R. Morel. Hemodynamics induced after acute reduction of proximal thoracic aorta compliance. Eur. J. Vasc. Endovasc. Surg. 26:195-204, 2003.

${ }^{6}$ Langewouters, G. J. Visco-elasticity of the human aorta in vitro in relation to pressure and age. Thesis, Free University Amsterdam, 1982.

${ }^{7}$ McDonald, D. A., W. W. Nichols, and M. F. O'Rourke. Blood Flow in Arteries. London: Edward Arnold, 1990.

${ }^{8}$ Mitchell, G. F. Impedance progress: aortic diameter rears its head again? Hypertension 49:1207-1209, 2007.

${ }^{9}$ Mitchell, G. F., P. R. Conlin, M. E. Dunlap, Y. Lacourciere, J. M. Arnold, R. I. Ogilvie, J. Neutel, J. L. Izzo, Jr., and M. A. Pfeffer. Aortic diameter, wall stiffness, and wave reflection in systolic hypertension. Hypertension 51:105-111, 2008.

${ }^{10}$ Murgo, J. P., N. Westerhof, J. P. Giolma, and S. A. Altobelli. Aortic input impedance in normal man: relationship to pressure wave forms. Circulation 62:105-116, 1980. 
${ }^{11}$ O'Rourke, M. F., and W. W. Nichols. Aortic diameter, aortic stiffness, and wave reflection increase with age and isolated systolic hypertension. Hypertension 45:652-658, 2005.

${ }^{12}$ Randall, O. S., G. C. van den Bos, and N. Westerhof. Systemic compliance: does it play a role in the genesis of essential hypertension? Cardiovasc. Res. 18:455-462, 1984.

${ }^{13}$ Reymond, P., Y. Bohraus, F. Perren, F. Lazeyras, and N. Stergiopulos. Validation of a patient-specific 1-D model of the systemic arterial tree. Am. J. Physiol. Heart Circ. Physiol. 301:H1173-H1182, 2011.

${ }^{14}$ Reymond, P., F. Merenda, F. Perren, D. Rufenacht, and N. Stergiopulos. Validation of a one-dimensional model of the systemic arterial tree. Am. J. Physiol. Heart Circ. Physiol. 297:H208-H222, 2009.

${ }^{15}$ Stergiopulos, N., J. J. Meister, and N. Westerhof. Simple and accurate way for estimating total and segmental arterial compliance: the pulse pressure method. Ann. Biomed. Eng. 22:392-397, 1994.

${ }^{16}$ Stergiopulos, N., P. Segers, and N. Westerhof. Use of pulse pressure method for estimating total arterial compliance in vivo. Am. J. Physiol. 276:H424-H428, 1999.

${ }^{17}$ Stergiopulos, N., and N. Westerhof. Determinants of pulse pressure. Hypertension 32:556-559, 1998.

${ }^{18}$ Vasan, R. S. Pathogenesis of elevated peripheral pulse pressure: some reflections and thinking forward. Hypertension 51:33-36, 2008.

${ }^{19}$ Westerhof, N., P. Sipkema, G. C. van den Bos, and G. Elzinga. Forward and backward waves in the arterial system. Cardiovasc. Res. 6:648-656, 1972.

${ }^{20}$ Wilmink, A. B., H. J. Pleumeekers, A. W. Hoes, C. S. Hubbard, D. E. Grobbee, and C. R. Quick. The infrarenal aortic diameter in relation to age: only part of the population in older age groups shows an increase. Eur. J. Vasc. Endovasc. Surg. 16:431-437, 1998. 